\title{
A LETTER TO THE EDITOR
}

\section{Carcinogenicity of Benzotrichloride Administered to Mice by Gastric Intubation}

\begin{abstract}
Epidemiological studies suggest that benzotrichloride (BTC) is a human carcinogen. In the present study, BTC was tested to evaluate its ability to induce lung tumors as a result of systemic exposure. Administration of BTC by gastric intubation, 2.0-0.0315 $\mu \mathrm{l} /$ mouse (4 doses), twice a week for 25 weeks, in female ICR mice, produced forestomach tumors (squamous cell caricinoma and papilloma), lung tumors (adenocarcinoma and adenoma) and tumors of the hematopoietic system (thymic lymphosarcoma and lymphatic leukemia) with dose-related response by 18 months. The present and previous studies indicate that the target organs of BTC carcinogenesis in mice are the local tissue which is primarily exposed, and the lung and hematopoietic tissue when BTC is administered systemically.
\end{abstract}

Key words: Carcinogenicity-Benzotrichloride-ICR mouse-Gastric intubation-Stomach cancer-Lung cancer

We have reported the occurrence of lung cancer among workers in factories where benzoyl chloride and chlorinated toiuenes are manufactured., ${ }^{1,2)}$ A mortality study in the UK also showed excess cancers of the respiratory tract and digestive system in workers exposed to benzoyl chloride or chlorinated toluenes. ${ }^{3)}$ We have reported that benzotrichloride (BTC) is very probably responsible for causing the lung cancer seen in benzoyl chloride manufacturing workers, based on mouse skin painting experiments ${ }^{4}$ in which a high incidence of skin cancer was observed in mice exposed to BTC. However, apart from the induction of skin cancers, we were interested in the development of lung tumors in mice exposed to BTC. Two possibilities were proposed for the induction of lung tumors. First, BTC vaporized from the skin is inhaled and might directly affect the lung. Second, BTC penetrates the skin and/or its metabolites might affect the lung. To elucidate the latter, indirect effect on the lungs, administration of BTC to mice by gastric intubation was performed with special reference to the dose-response relationship.

Reagent-grade BTC was purchased from Tokyo Kasei Co. Ltd., Tokyo, and used without further purification. Gas chromatographic analysis of the chemical verified $99.5 \%$ purity.

Specific-pathogen-free (SPF) female ICR mice, 4 weeks old, were obtained from Japan SLC, Inc. (formerly Shizuoka Agricultural Co-operative for Experimental Animals), Hamamatsu, and 10 animals were housed in each polycarbonate cage with wood-chip bedding in a room supplied with all-fresh air and main- 
tained at $24 \pm 1{ }^{\circ} \mathrm{C}$ and $55 \pm 5 \%$ relative humidity. Tap water pasteurized with ultraviolet rays and autoclaved laboratory feed (CE-2; CLEA Japan Inc., Tokyo), were provided ad libitum. Cages and bedding were autoclaved before use.

Two hundred 9-week-old ICR female mice were randomly divided into 5 groups (40 mice/group). BTC was dissolved in $0.1 \mathrm{ml}$ of sterilized sesame oil and doses of $2 \mu 1$ (group A), $0.5 \mu 1$ (group B), $0.125 \mu 1$ (group C), $0.0315 \mu 1$ (group D) and $0 \mu 1$ (control) wre administered to mice twice a week for 25 weeks. The experiment was terminated at 18th months after the start of administration. The mice in group $\mathrm{A}$ were killed at 12 months because of their high mortality (95\%) and the poor condition of the surviving mice. Moribund and surviving mice were killed with ether. After gross observation, organs and tumors were dissected. Tissues were fixed in 10\% phosphate-buffered formalin and embedded in paraffin, sectioned $3 \mu \mathrm{m}$ thick and stained with hematoxylin and eosin. Other special stainings were used when needed.

The mortality of the treated mice increased dose-dependently. The mice in group A died earlier than those in the other groups. The cause of death was mostly thymic lymphosarcoma and stomach cancer. The time to $50 \%$ mortality were 6.5 months and 16.5 months in group A and group B, respectively. Mortality of the mice in groups $\mathrm{C}$ and $\mathrm{D}$ did not reach $50 \%$ even by 18 months (Fig. 1).

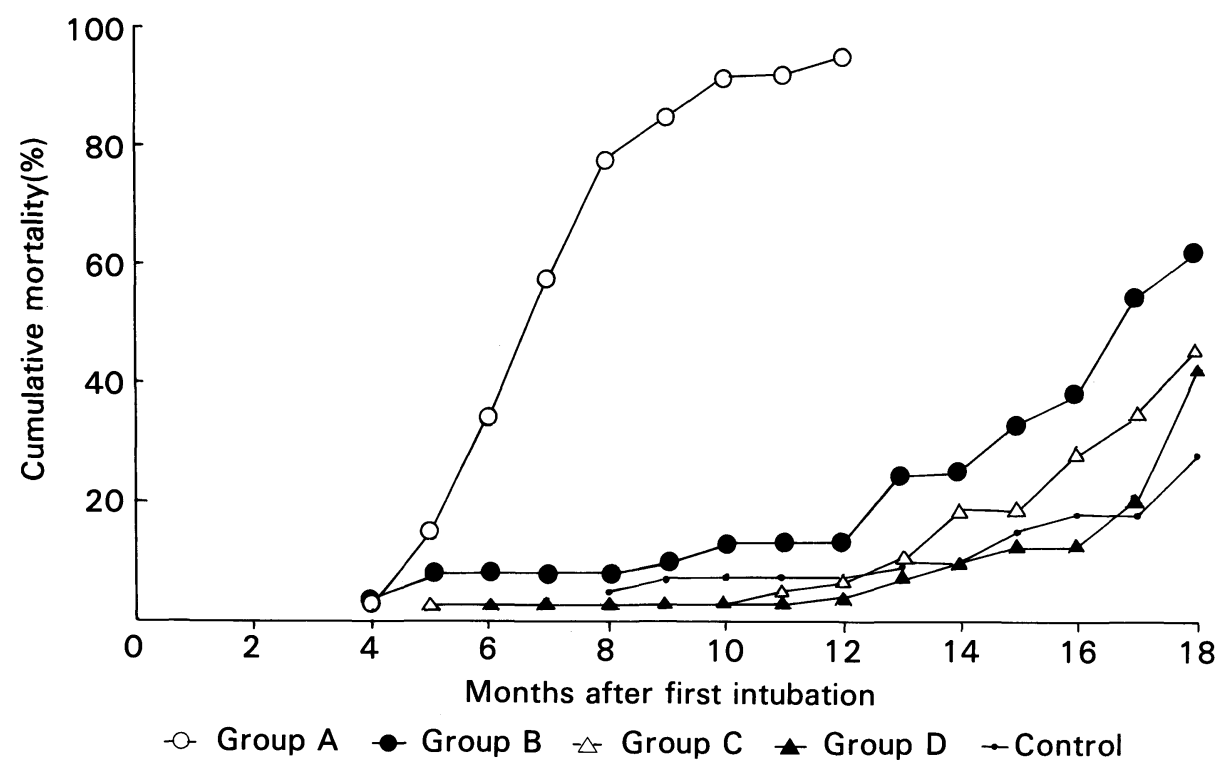

Fig. 1. Mortality of the Mice Exposed to Benzotrichloride by Intubation. 
other cell types were observed. Lung tumors in the dosed groups were mostly multicentric.

The incidence of hematopoietic tumors in group A was significantly higher than in the control. Thymic lymphosarcoma was the earliest tumor observed tumors in each group, as was seen in the case of skin exposure ${ }^{4)}$. The incidence of thymic lymphosarcoma was $18 \%(7 / 38)$ and $5 \%(2 / 40)$ in groups A and B, respectively, by 6 months. The other hematopoietic tumor was lymphatic leukemia. The rather low incidences of hematopoietic tumors may be attributable to the low dose used.

The Cochran-Armitage statistical test demonstrated a positive trend in the incidence of tumors of the forestomach, lung and hematopoietic system. Other neoplasms occurred sporadically among the groups at low incidences (3\%-8\%). They consisted of mammary gland adenomas or carcinoma, salivary gland carcinoma, Harderian gland carcinoma and liver hemangioendothelioma. The incidence of these tumors was not statistically significant, however, statistical analysis by the Cochran-Armitage trend test showed a positive dose-related trend $(\mathrm{p}<$ 0.05 ) for the incidence of mammary tumors.

The present study demonstrated that gastric administration of BTC produces stomach tumors, lung tumors and tumors of the hematopoietic system in a dose-related manner. Stoner et al. reported that intraperitoneal injection of BTC produced a significant increase in the lung tumor response in strain $\mathrm{A} / \mathrm{J}$ mice within 24 weeks $^{5}$. The possibility of lung tumor production by extra-pulmonary exposure of BTC was verified in the present experiment together with the results of strain A mouse lung bioassay. BTC has been shown to have potent mutagenic activity with or without metabolic activation in the bacterial reversion test system $^{6,7)}$. Therefore, after being absorbed from the stomach, BTC may be metabolized in the liver and/or in the lungs, and BTC metabolites may then exert tumorigenic activity in the lungs. BTC is a direct alkylating agent ${ }^{4)}$ and reacts strongly with ${ }^{32} \mathrm{P}$-labelled DNA in vitro ${ }^{8}$. As in the skin painting experiments ${ }^{4)}$, BTC displayed local carcinogenicity when administered intragastrically. The carcinogenicity and mutagenicity data accompanied by chemical reactivity in vitro suggest that BTC has both the properties of a direct carcinogen and a procarcinogen. The present and previous studies indicate that the target organ of BTC carcinogenesis in mice is the local tissue which is primarily exposed, and the lung and hematopoietic tissue when administered systemically.

\section{REFERENCES}

1) Sakabe H, Fukuda K. An updating report on cancer among benzoyl chloride manufacturing workers. Ind. Health $1977 ; 15: 173-4$.

2) Sakabe H, Matsushita H, Koshi S. Cancer among benzoyl chloride manufacturing workers. Ann. N.Y. Acad. Sci. 1976; 271: 67-70

3) Sorahan T, Waterhouse JAH, Cooke MA, Smith EMB, Jackson JR, Temkin L. A mortality 
study of workers in a factory manufacturing chlorinated toluenes. Ann. occup. Hyg. 1983; 27 : 173-82.

4) Fukuda K, Matsushita H, Sakabe H, Takemoto K. Carcinogenicity of benzyl chloride, benzal chloride, benzotrichloride and benzoyl chloride in mice by skin application. Gann 1981; 72: 65564.

5) Stoner GD, You M, Morgan MA, Superczenski MJ. Lung tumor induction in strain A mice with benzotrichloride. Cancer Letters 1986; 33: 167-73.

6) Yasuo K, Fujimoto S, Katoh M, Kikuchi Y, Kada T. Mutagenicity of benzotrichloride and related compounds. Mutation Res. 1978; 58: 143-50.

7) Matsushita H, Gotoh S, Hamano Y. A mutagenicity testing method for volatile compounds. Proc. of the 54th Annual Meeting of Japan Association of Industrial Health, 1981: 280-1.

8) Kawanishi S, Kurasako K, Inoue S, Seki Y, Inui S, Sano S. Predicting carcinogenicity of halogenated hydrocarbons by their reactivity with DNA. Proc. of the 58th Annual Meeting of Japan Association of Industrial Health, 1985: 94-5.

1) National Institute of Industrial Health, 21-1, Nagao 6-chome, Tama-ku, Kawasaki 214, Japan

Kazuo FUKUDA ${ }^{1)}$

Hidetsuru MATSUSHITA ${ }^{2)}$

2) Graduate School of Nutritional and Environmental Sciences, University of Shizuoka, Kazuo TAKEMOTO ${ }^{3)}$ and Tadao TOYA ${ }^{1)}$

Yada, 52-1, Shizuoka City, Shizuoka 422, Japan

3) Department of Public Health, Saitama Medical School, Hongo 38, Moroyama-cho, Iruma-gun, Saitama 350-14, Japan 